\title{
Clinicopathological Characteristics of LgG4-Related Lung Disease
}

\author{
Jia Liu \\ Sun Yat-sen Memorial Hospital, Sun Yat-sen University \\ Yuxiang Liu \\ Sun Yat-sen Memorial Hospital, Sun Yat-sen University \\ Ximing Shen \\ Sun Yat-sen Memorial Hospital, Sun Yat-sen University \\ Zhanghai He \\ Sun Yat-sen Memorial Hospital, Sun Yat-sen University \\ Tingfeng Yu \\ Sun Yat-sen Memorial Hospital, Sun Yat-sen University \\ Li Pang \\ Sun Yat-sen Memorial Hospital, Sun Yat-sen University

\section{Xiaoyan Jin} \\ Sun Yat-sen Memorial Hospital, Sun Yat-sen University \\ Lingyun Wang ( $\square$ wanglyun@mail.sysu.edu.cn ) \\ Sun Yat-sen Memorial Hospital, Sun Yat-sen University
}

\section{Research Article}

Keywords: Immunoglobulin G4-related disease, Immunoglobulin G4-related lung disease, Clinical characteristics, Pathology, Chest computed tomography

Posted Date: November 8th, 2021

DOI: https://doi.org/10.21203/rs.3.rs-1021258/v1

License: (c) (1) This work is licensed under a Creative Commons Attribution 4.0 International License. Read Full License 


\begin{abstract}
Background: Immunoglobulin G4-related lung disease (IgG4-RLD) is a rare entity. The aim of this study is to retrospectively analyzed the clinicopathological characteristics of IgG4-RLD pathologically confirmed in order to improve the diagnosis and treatment of the disease.
\end{abstract}

Methods: We screened the pathological reports of 4838 patients with pulmonary surgery and/or biopsy specimens at Sun Yat-sen Memorial Hospital affiliated to Sun Yat-sen University from April 2017 to April 2021, and 65 patients with suspected IgG4-RLD had an immunohistochemical staining for IgG4 and IgG. Finally, 10 patients with definite IgG4-RLD pathologically comfirmed were enrolled and analyzed.

Results: The incidence of IgG4-RLD pathologically confirmed was $0.2 \%$ (10/4838). Ten patients had a mean age of 59.7 years at diagnosis with a male: female ratio of 9:1. The initial clinical manifestations were lack of specificity and cough was the most common symptom (4/10). More than one organ was involved in most patients (8/10) and lymph node involvement was often seen (7/10). Serum IgG4 were detected and elevated in 6 patients. The levels of serum tumor markers were within normal range or slightly elevated. The chest computed tomography (CT) and/or ${ }^{18} \mathrm{~F}$-fluorodeoxyglucose positron emission tomography-computed tomography ( ${ }^{18} \mathrm{~F}$-FDG PET-CT) findings showed 5 patients of mix type, 3 patients of solid nodular type and 2 patients of bronchovascular type. All pulmonary mass and large nodules with solid pattern had spiculated margins and inhomogeneous enhancement with or without pleural indentation and lobulated appearance. Abundant lymphoplasmacytic infiltration and fibrosis were observed in all patients. The expressions of IgG4 and IgG in the plasma cells were up-regulated in the immunostained sections of lung. Seven patients received prednisone with or without additional immunosuppressive drugs and responded well.

Conclusions: Our study suggests that multiple imaging findings combined with elevated serum IgG4 as well as serum tumor markers without significant increase could provide diagnostic support for IgG4-RLD.

\title{
Introduction
}

Immunoglobulin G4-related disease (IgG4-RD) is a recently recognized systemic fibro-inflammatory disease characterized by pathologically dense lymphoplasmacytic infiltration, storiform fibrosis and obliterative phlebitis [1]. This disease can affect almost any organ, such as pancreas, hepatobiliary system, salivary and lacrimal glands, kidney and retroperitoneum [2]. IgG4-related lung disease (IgG4-RLD) was first reported in 2004 [3]. To the best of our knowledge, the literature on IgG4-RLD is limited to single case reports and small case series and the incidence of IgG4-RLD has not been reported. What's more, most of them have not been pathologically confirmed by lung tissue. Other causes, such as pulmonary infection and congestion may also lead to pulmonary lesions of IgG4-RD, which may be mistaken for IgG4-RLD. Therefore, clinical analysis of IgG4-RLD based on pathological diagnosis may lead to a more accurate understanding of the entity. The present study was to retrospectively analyze the clinicopathological characteristics of IgG4-RLD which was verified by pathological diagnosis for the purpose of improving the diagnosis and treatment of IgG4-RLD.

\section{Methods}

Patients. The retrospective study was conducted at Sun Yat-sen Memorial Hospital affiliated to Sun Yat-sen University. We reviewed the pathological reports of 4838 patients who underwent lung resection and/or biopsy between April 2017 and April 2021 using the electronic pathological system. Among them, immunohistochemical staining for IgG and IgG4 was performed in 65 patients with suspected IgG4-RLD. According to the 2012 international consensus on the pathology of IgG4-RD [4] described briefly below, 10 patients were pathologically diagnosed as IgG4-RLD, and finally 6 patients had a histologically highly suggestive diagnosis and 4 patients had a probable histological diagnosis of IgG4-RD. The flow diagram of the screening and diagnostic process is exhibited in Fig. 1. Of the 10 patients, 4 patients (case 1-4) received pulmonary lobectomy via video-assisted thoracoscopic surgery (VATS) because of a suspicion of lung cancer, 1 patients (case 5) with open lung biopsy (OLB), 1 patients (case 6) with percutaneous lung biopsy (PLB) and 4 patients (case 7-10) with transbronchial lung biopsy (TLB). Patients with malignant tumors, lymphoproliferative disorder, Castleman's disease and sarcoidosis were excluded. Once cases were identified, relevant clinical data were extracted from the electronic medical record. The clinical, serological, radiological and histopathological features, treatment and responses of the patients were collected and analyzed retrospectively. The present study was conducted in conformity with the Declaration of Helsinki and was approved by the Ethics Committee of Sun Yat-sen Memorial Hospital, Sun Yat-sen University (number, SYSEC-KY-KS-2021225). Informed consent was exempt because of the retrospective nature of the study.

\section{Pathological Diagnosis Criteria Of Igg4-rld}

The pathological diagnostic criteria were based on the 2012 international consensus on the pathology of IgG4-RD [4]. The diagnostic criteria are based primarily on the histological features, such as dense lymphoplasmacytic infiltration, fibrosis, usually storiform fibrosis in character and obliterative phlebitis. The number of IgG4+ plasma cells and IgG4+/lgG+ plasma cell ratio in the tissue are secondary in importance. Three categories were proposed for pathological diagnosis: histologically highly suggestive diagnosis of IgG4-RLD, probable histological features of IgG4-RLD and insufficient histopathological evidence of IgG4-RLD listed below. Patients with the two categories of histologically highly suggestive and probable histological features of the disease were included in the current study.

1. Histologically highly suggestive diagnosis of IgG4-RLD:

1. $\geq 2$ items of histological features.

2. IgG4+ cells $>50$ cells/high-power field (HPF).

3. $\operatorname{lgG} 4+/ \lg G+$ plasma cell ratio $>40 \%$. 
2. Probable histological features of IgG4-RLD:

1. at least 1 item of histological features.

2. IgG4+ cells $>50$ cells/HPF from lung surgical specimen or $>20$ cells/HPF from biopsy, or $\lg 4+/ \lg$ + ratio $>40 \%$.

This category requires additional evidence to confirm the diagnosis: serum IgG4 concentration $>2.01 \mathrm{~g} / \mathrm{l}$ according to the detective range of our hospital or other organ involvement demonstrated by imaging or pathological finding.

3. Insufficient histopathological evidence of IgG4-RLD: no conforming to the above two categories.

Histopathological studies. The hematoxylin and eosin (H\&E) staining and immunohistochemical staining for IgG4 and IgG were performed in the Department of Pathology, Sun Yat-sen Memorial Hospital. The stained sections were independently reassessed by two investigators (Ximing Shen and Zhanghai He). First, the immunostained sections were scanned with a light microscope at low-power magnification $(\times 40)$ to identify three different hot spots of IgG4+ plasma cells. Then the IgG4+ plasma cells number was performed in the three fields using HPF $(\times 400)$ and taken the average for each investigator. The same three fields at should be counted for the lgG+ plasma cells and then $\mathrm{lgG} 4+/ \mathrm{lgG}+$ plasma cell ratio was calculated. The numbers of the two researchers averaged as the final results respectively. However, when significantly different counts for a section occurred, a double-headed microscope was used to reach an agreement.

Imaging. All included patients received chest computed tomography (CT) and/or ${ }^{18} \mathrm{~F}$-fluorodeoxyglucose positron emission tomography-computed tomography $\left({ }^{18} \mathrm{~F}\right.$-FDG PET-CT) at baseline. These images were reviewed by a professional radiologist (Yuxiang Liu). According to the predominant radiological abnormality on CT examination, patients were divided into five types: solid nodular, round ground-glass opacity (GGO), bronchovascular, alveolar interstitial, and alveolar consolidative type, which were mainly proposed by Inoue et al [5]. Solid nodular type was characterized by a solitary nodule or mass; round GGO type by multiple round-shaped GGOs; alveolar interstitial type by honeycombing, bronchiectasis, and diffuse GGO; bronchovascular type by thickening of bronchovascular bundles and interlobular septa; alveolar consolidative type by airspace filling opacities obscuring vasculature in a segmental or lobar distribution $[6,7,8]$. When two or more types coexisted, they were classified as mixed type. Mass was defined as a lung solid lesion larger than $3 \mathrm{~cm}$ in diameter; large nodule, $1 \mathrm{~cm} \leq$ diameter $\leq 3 \mathrm{~cm}$; small nodule, diameter $<1 \mathrm{~cm}$ [9].

Follow-up. The last follow-up ended in October 2021. The 10 patients with IgG4-RLD had a complete follow-up with a mean of 27 months (range 6-54 months). The clinical, laboratory and imaging data of the patients were recorded in detail at each follow-up visit.

\section{Results}

\section{Clinical and laboratory features}

The clinical and laboratory features of the 10 patients IgG4-RLD are summarized in Table 1. The mean age of the patients was 59.7 years at diagnosis with a male: female ratio of 9:1. The time from onset to diagnosis averaged 26.5 months (range 0.2-120.0 months). Out of the 10 patients, 1 patient had concomitant chronic obstructive pulmonary disease and 3 patients had a history of tuberculosis which had been cured. And 6 patients had a history of smoking. The most common initial symptom was cough (4/10, 40\%). Other symptoms included chest or back pain (2/10, 20\%), hemoptysis (2/10, 20\%), lowgrade fever $(1 / 10,10 \%)$ and ocular symptoms $(1 / 10,10 \%)$. Three patients were initially asymptomatic and incidentally identified by abnormal pulmonary changes on chest radiological examinations $(3 / 10,30 \%)$. The majority of the patients $(8 / 10,80 \%)$ had multiple organs involved (mean number 2.4 , range $1-6)$. Mediastinal/hilar lymph node was the most commonly involved extrapulmonary organ (7/10, 70\%), followed by nasal sinus (3/10, 30\%), lacrimal gland (1/10, $10 \%)$, parotid gland $(1 / 10,10 \%)$, submandibular gland $(1 / 10,10 \%)$ and pituitary gland $(1 / 10,10 \%)$. Serum IgG4 were detected and elevated in 6 patients $>$ $2.01 \mathrm{~g} / \mathrm{l})$, while 4 patients had not the results because of discharge from the hospital. Serum erythrocyte sedimentation rate (ESR) level was elevated in 9 of the 10 patients $(>15 \mathrm{~mm} / \mathrm{h}$ ) and high sensitive C-reaction protein (hs-CRP) level was elevated in 6 of the 10 patients (>3 mg/l). Of the 10 patients, 3 patients had a slightly elevated level of serum cytokeratin fraction 21-1 (CYFRA 21-1) (> 3.3 ng/ml) and 1 patients with a slightly elevated level of serum neuronspecific enolase (NSE) (> $16.3 \mathrm{ng} / \mathrm{ml}$ ), while serum carcinoembryonic antigen (CEA) and cancer antigen 125 (CA125) levels of all patients were in the normal range. 
Table 1

Clinicopathological features of 10 patients with IgG4-related lung disease

\begin{tabular}{|c|c|c|c|c|c|c|c|c|c|c|c|c|c|}
\hline \multirow{2}{*}{$\begin{array}{l}\text { Case } \\
\text { No. }\end{array}$} & \multirow{2}{*}{$\begin{array}{l}\text { Age } \\
\text { (years) }\end{array}$} & \multirow[t]{2}{*}{ Gender } & \multirow{2}{*}{$\begin{array}{l}\text { Diagnostic } \\
\text { approach }\end{array}$} & \multicolumn{3}{|c|}{ Histopathological features } & \multicolumn{7}{|c|}{ Serological findings } \\
\hline & & & & $\begin{array}{l}\lg G 4+ \\
\text { (/HPF) }\end{array}$ & $\begin{array}{l}\text { lgG+ } \\
(/ \mathrm{HPF})\end{array}$ & $\begin{array}{l}\lg G 4+/ \lg G+ \\
(\%)\end{array}$ & $\begin{array}{l}\lg G 4 \\
(g / l)\end{array}$ & $\begin{array}{l}\text { ESR } \\
(\mathrm{mm} / \mathrm{h})\end{array}$ & $\begin{array}{l}\text { hs-CRP } \\
\text { (mg/l) }\end{array}$ & $\begin{array}{l}\text { NSE } \\
(\mathrm{ng} / \mathrm{ml})\end{array}$ & $\begin{array}{l}\text { CYFRA 21-1 } \\
(\mathrm{ng} / \mathrm{ml})\end{array}$ & $\begin{array}{l}\text { CEA } \\
(\mathrm{ng} / \mathrm{ml})\end{array}$ & $\begin{array}{l}\text { CA125 } \\
(\mathrm{U} / \mathrm{ml})\end{array}$ \\
\hline 1 & 65 & $\mathrm{~F}$ & VATS & 97 & 214 & 45.3 & NA & 8 & 1.2 & 17.2 & 3.1 & 1.9 & 10.6 \\
\hline 2 & 50 & M & VATS & 78 & 175 & 44.6 & NA & 20 & 0.3 & 10.4 & 3.5 & 1.9 & 6.9 \\
\hline 3 & 58 & M & VATS & 52 & 130 & 40.0 & NA & 18 & 1.0 & 10.7 & 4.1 & 1.5 & 1.5 \\
\hline 4 & 64 & M & VATS & 56 & 129 & 43.4 & NA & 61 & 9.5 & 8.4 & 3.8 & 2.4 & 29.3 \\
\hline 5 & 58 & M & OLB & 65 & 151 & 43.0 & 4.6 & 101 & 156.8 & 9.3 & 1.8 & 1.4 & 13.3 \\
\hline 6 & 58 & $M$ & PLB & 29 & 112 & 25.9 & 11.7 & 95 & 117.3 & 7.7 & 2.5 & 2.5 & 10.1 \\
\hline 7 & 64 & $M$ & TLB & 43 & 195 & 22.1 & 3.3 & 77 & 49.7 & 11.0 & 1.7 & 1.2 & 9.5 \\
\hline 8 & 56 & $M$ & TLB & 122 & 248 & 49.2 & 8.6 & 52 & 23.3 & 10.7 & 2.7 & 1.1 & 10.0 \\
\hline 9 & 67 & $M$ & TLB & 34 & 113 & 30.1 & 7.3 & 87 & 25.8 & 10.6 & 2.3 & 0.9 & 18.7 \\
\hline 10 & 57 & $M$ & TLB & 40 & 62 & 64.5 & 56.3 & 92 & 3.0 & 8.2 & 2.1 & 0.7 & 17.3 \\
\hline
\end{tabular}

\section{Imaging Characteristics}

The pulmonary imaging characteristics of the 10 patients are summarized in Table 2. The representative images are shown in Fig. 2. The chest CT and/or ${ }^{18} \mathrm{~F}-$ FDG PET-CT findings showed 5 patients of mix type, 3 patients of solid nodular type and 2 patients of bronchovascular type. Eight patients had pulmonary mass and/or nodules with solid patterns. Of the 8 patients, 3 patients had single lung mass and 5 patients had large lung nodules, while all had multiple small nodules mostly scattered in all lobes. In addition to lobulated shape in 1 patients and pleural indentation in 4 patients, these pulmonary masses and large nodules had spicules of margin and heterogeneous density. Pulmonary nodules were bilateral in 6 patients except for 2 patients with lesions confined to the right lung. The features of mass or nodules of the 8 patients are summarized in Table 3 . Thickening of bronchovascular bundle, thickening of interlobular septa, thickening of pleura, round-shaped GGO, diffuse GGO, alveolar consolidation, bronchiectasia and enlarged mediastinal/hilar lymph node were detected in 5, 1, 4, 2, 1, 1, 4 and 7 patients, respectively. Nodular calcifications occurred in only 1 patient. Honeycombing, pleural ffusion and cysts were not observed in all patients.

Table 2

Imaging characteristics of 10 patients with IgG4-related lung disease

\begin{tabular}{|c|c|c|c|c|c|c|c|c|c|c|}
\hline $\begin{array}{l}\text { Case } \\
\text { No. }\end{array}$ & Mass/nodule & $\begin{array}{l}\text { Thickening of } \\
\text { bronchovascular } \\
\text { bundle }\end{array}$ & $\begin{array}{l}\text { Thickening } \\
\text { of } \\
\text { interlobular } \\
\text { septa }\end{array}$ & $\begin{array}{l}\text { Round- } \\
\text { shaped } \\
\text { GGO }\end{array}$ & $\begin{array}{l}\text { Diffuse } \\
\text { GGO }\end{array}$ & Bronchiectasia & $\begin{array}{l}\text { Thickening } \\
\text { of pleura }\end{array}$ & $\begin{array}{l}\text { Alveolar } \\
\text { consolidation }\end{array}$ & $\begin{array}{l}\text { Enlarged } \\
\text { mediastinal } \\
\text { /hilar } \\
\text { lymph node }\end{array}$ & Type \\
\hline 1 & + & & & & & & & & & Solid nod \\
\hline 2 & + & + & & + & & + & & & + & Mix \\
\hline 3 & + & + & & & & + & & & & Mix \\
\hline 4 & + & + & & + & & + & + & & & Mix \\
\hline 5 & + & & & & & & & & + & Solid nod \\
\hline 6 & + & & + & & & & + & + & + & Mix \\
\hline 7 & + & & & & & & & & + & Solid nod \\
\hline 8 & + & & & & + & & & & + & Mix \\
\hline 9 & & + & & & & & + & & + & Bronchov \\
\hline 10 & & + & & & & + & + & & + & Bronchov \\
\hline
\end{tabular}


Table 3

Imaging features of pulmonary mass or nodules in 8 patients with IgG4-related lung disease

\begin{tabular}{|c|c|c|c|c|c|c|c|c|c|}
\hline $\begin{array}{l}\text { Case } \\
\text { No. }\end{array}$ & Mass & Larnodule & $\begin{array}{l}\text { Small } \\
\text { nodule }\end{array}$ & Solid & Spiculated margin & $\begin{array}{l}\text { Lobulated } \\
\text { shape }\end{array}$ & Pleural indentation & Heterogeneous enhancement & Location \\
\hline 1 & & + & + & + & + & & & + & All lobes \\
\hline 2 & & + & + & + & + & & & + & $\begin{array}{l}\text { Right } \\
\text { upper } \\
\text { and } \\
\text { lower } \\
\text { lobe }\end{array}$ \\
\hline 3 & & + & + & + & + & & + & + & All lobes \\
\hline 4 & + & & + & + & + & & + & + & All lobes \\
\hline 5 & + & & + & + & + & & & + & All lobes \\
\hline 6 & & + & + & + & + & & & + & All lobes \\
\hline 7 & & + & + & + & + & & + & + & All lobes \\
\hline 8 & + & & & + & + & + & + & + & $\begin{array}{l}\text { Right } \\
\text { upper } \\
\text { lobe }\end{array}$ \\
\hline
\end{tabular}

\section{Pathological Findings}

The incidence of IgG4-RLD pathologically confirmed was $0.2 \%$ (10/4838). All lung tissue specimens from the 10 patients with pathologically confirmed IgG4RLD are available. Most of the lung specimens were gray-white or gray-yellow. The H\&E staining revealed chronic pulmonary inflammation: dense lymphoplasmic cells infiltration $(10 / 10,100 \%)$ and fibrosis $(10 / 10,100 \%)$. The representative images are shown in Figure 2 . No malignancy or dysplasia was found in all patients with IgG4-RLD. The immunohistochemistry staining sections for IgG4 and IgG of the 10 patients were carefully reviewed. The results demonstrated that IgG4 and IgG in the plasma cells were highly expressed in the lgG4-RLD sections. Meanwhile, IgG4+/lgG+ plasma cell ratio was performed in the IgG4-RLD tissues. The data are shown in Table 1 and the representative images are shown in Fig. 3.

\section{Therapy and responses}

Seven patients received prednisone with or without additional immunosuppressive drugs: 5 with prednisone only, 2 with prednisone and cyclophosphamide. Two patient underwent lung surgery did not use prednisone or immunosuppressive drugs. One patients with mild disease received symptomatic therapy only. Symptoms improved in all patients after treatment before they were discharged from hospitals. All patients came for several follow-up visits as required. The levels of serum IgG4 decreased gradually in 6 patients with initially elevated serum IgG4, while 1 patients had increased serum IgG4 again during prednisone reduction. The follow-up chest CT were performed in all patients. Six patients had improved chest CT with nodules or mass shrinkage. Two patients had no change in pulmonary nodules. Two patients relapsed and had new pulmonary nodules, then they disappeared after treatment with sufficient prednisone again combined with immunosuppressive drugs.

\section{Discussion}

IgG4-RLD is a rare condition which is still not well understood and the incidence of IgG4-RLD is lacking. Our study showed the incidence of IgG4-RLD pathologically confirmed was $0.2 \%$ in our hospital. In the present study, we summarized and analyzed the clinicopathological characteristics of the 10 patients with IgG4-RLD pathologically confirmed based on a single-institution experience.

In our study, there were several important findings: (i) Elevated serum IgG4 was observed in the patients detected and serum tumor markers had no significant elevation in the patients with IgG4-RLD; (ii) Clinical manifestations and radiological findings were nonspecific. Some patients were initially asymptomatic and pulmonary lesions were accidentally identified by chest imaging examination. Moreover, on account of that the imaging often mimics pulmonary malignancy, patients with IgG4-RLD might be misdiagnosed as lung cancer and undergo unnecessary lung resection. Thus, when lung is independently affected or combined with special anatomical site involved which is difficult to help make a definite diagnosis, the diagnosis of IgG4-RLD is challenging, and a brief strategy for combining radiological findings with serum IgG4 and tumor markers was suggested to differentiate IgG4-RLD from lung cancer; (iii) Most patients responded well to prednisone with or without additional immunosuppressive drugs.

The clinical symptoms of IgG4-RLD are nonspecific mainly depending on the location of pulmonary lesions. Our study showed that the initial respiratory symptoms had cough, chest or back pain, hemoptysis and low-grade fever. And pulmonary abnormalities were occasionally found by chest radiological examination in the asymptomatic patients. This phenomenon has been reported to various extent in the previous studies [5-11].

In 2009, Inoue and colleagues proposed four major subtypes on the basis of the predominant radiologic abnormality: solid nodular, round-shaped GGO, alveolar interstitial and bronchovascular type [5]. In addition, additional alveolar consolidation type was observed as the fifth type of imaging classification [6-8]. In present study, pulmonary mass and nodules were commonly seen and it was noteworthy that most were associated with the manifestations of other types, such as thickening of bronchovascular bundle, thickening of interlobular septa, bronchiectasia, round-shaped GGO, diffuse GGO and consolidation. It 
was not easy to classify them as one of the above five types. Therefore, we divided these patients into mix type when two or more types occurred together. What's more, we found all pulmonary mass and large nodules with solid pattern had spiculated margins and inhomogeneous enhancement with or without pleural indentation and lobulated appearance, which were usually indicative of malignancy [12, 13]. These overlapping CT characteristics of IgG4-RLD and lung cancer suggested that they might share some pathological bases. For lung cancer, the appearance can be explained by contraction of internal fibrosis, different growth rates of cancerous cells and extension of malignant cells along the lung interstitium [14]. Inoue et al demonstrated that the pathological findings, such as fibrosis and diffuse lymphoplasmacytic infiltration along the interlobular septa corresponded to these radiologic findings in IgG4-RLD [5], which was also discovered in our study. We speculated that the imaging manifestations of IgG4-RLD resembling lung cancer might be due to shared fibrosis and dense lymphoplasmacytic infiltration simulating the pattern of cancerous invasion. Thus, it is a great challenge to identify IgG4-RLD with pulmonary mass and/or large nodules from lung cancer.

To solve this problem, we should focus on the following points: (i) Beside pulmonary mass and/or large nodules, attention should be paid to the radiological manifestations of other subtypes as mentioned above, which may provide some support for the diagnosis of IgG4-RLD; (ii) In such cases, serum IgG4 should be measured. Serum IgG4 has highly diagnostic value for IgG4-RD and was positively correlated with the number of organs involvedl [15]. Wang and colleague reported $84 \%$ patients with IgG4-RLD had an elevated serum IgG4 concentration [16]; (iii) For patients with initially suspected lung cancer, traditional serum biomarkers are usually measured as a routine examination for the early diagnosis of lung cancer. Several studies have demonstrated the importance of serum tumor markers in diagnosis of lung cancer [17-20]. They investigated that the levels of serum tumor markers, such as NSE, CYFRA 21-1, CEA, CA125 are significantly higher in lung cancer than benign lung diseases and the various biomarker panels can increase the sensitivity and diagnostic accuracy in lung cancer. Our study showed all patients with IgG4-RLD had a normal concentration or only a slight increase of serum biomarkers, while serum IgG4 was elevated in all patients detected. However, serum IgG4 is not routinely examined in many hospitals until now. It is not economically feasible to measure serum IgG4 in all patients with pulmonary abnormalities. Therefore, for patients with initially suspected lung cancer, if the imaging finding has multiple different manifestations of lung lesions, such as mass or nodules, bronchovascular bundle thickening, interlobular septa thickening, pleural thickening, bronchiectasia, round-shaped GGO, diffuse GGO and consolidation, and the levels of NSE, CYFRA 21-1, CEA, CA125 are within normal limits or slightly elevated, serum IgG4 should be measured. Elevated IgG4 can offer diagnostic support for IgG4-RLD and then experimental steroid therapy can be used to further confirm the diagnosis. When serum IgG4 is not elevated and lung cancer is strongly suspected, a lung biopsy can be used to establish the diagnosis. Interesting, our study showed elevated ESR and hs-CRP in most patients, which might reflect acute inflammation in IgG4-RLD.

Glucocorticoids are commonly used as the first-line treatment of IgG4-RD and most patients response well to this intervention. However, there is currently no universal consensus on the duration and tapering regimens of glucocorticoids. Glucocorticoids can combine with immunosuppressive drugs as first-line treatment when predictors of relapse are present, such as multiple organ injury, elevated serum IgG4 and immunoglobulin E (IgE) and peripheral eosinophilia [21]. Consistent with previous reports [7, 9] our study demonstrated the effectiveness of glucocorticoids with or without immunosuppressive drugs in IgG4RLD. Retreatment with glucocorticoids in combination with immunosuppressive drugs still showed significant improvement in the patient with relapses. Up to the last follow-up, 1 patients with symptomatic therapy only remained in a stable condition, which has been reported in Sun's study [7]. This phenomenon suggested that some patients might have self-limiting IgG4-RLD. However, little is known about the natural history of IgG4-RLD because the follow-up of studies was not long enough. In any case, long-term regular follow-up is essential for IgG4-RLD.

The strength of our study is that to our knowledge, we first reported the incidence of IgG4-RLD pathologically confirmed. Furthermore, we analyzed the clinicopathological characteristics of the patients with definite IgG4-RLD based on histopathological diagnosis and a brief approach to differentiate IgG4-RLD from lung cancer was proposed. However, the present study has some limitations. First, the generalizability of our findings may be questioned because the nature of this study is a single-center retrospective study with just a small amount of cases and the presence of selection bias. Large-scale prospective studies are needed for further confirmation. Second, our follow-up time is short and the whole course of IgG4-RLD can not be well investigated.

\section{Conclusions}

IgG4-RLD is a rare disorder and often mimics lung cancer. For clinicians, it is a great challenge to distinguish IgG4-RLD from lung cancer. This study analyzed the clinical characteristics of 10 patients with IgG4-RLD confirmed pathologically in our hospital. Our study suggested that multiple findings in imaging combined with elevated serum IgG4 as well as serum tumor markers without significant increase could provide diagnostic support for IgG4-RLD, and subsequent experimental steroid therapy could be performed to further confirm the diagnosis. If not, a lung biopsy was performed to make a definitive diagnosis.

\section{Abbreviations}

${ }^{18}$ F-FDG PET-CT: ${ }^{18}$ F-fluorodeoxyglucose positron emission tomography-computed tomography; CA125: cancer antigen 125; CEA: carcinoembryonic antigen; CT: computed tomography; CYFRA 21-1: cytokeratin fraction 21-1; ESR: erythrocyte sedimentation rate; F: female; GGO: ground-glass opacity; H\&E: hematoxylin and eosin; HPF: high power field; hs-CRP: high-sensitivity C-reactive protein; IgE: immunoglobulin E; IgG: immunoglobulin G; IgG4: immunoglobulin G4; IgG4-RD: immunoglobulin G4-related disease; IgG4-RLD: immunoglobulin G4-related lung disease; M: male; NA:data not available; NSE: neuron-specific enolase; OLB: open lung biopsy; PLB: percutaneous lung biopsy; TLB: transbronchial lung biopsy; VATS: video-assisted thoracoscopic surgery.

\section{Declarations}

\section{Funding}

The present study was not funded by any grants. 


\section{Conflicts of interest}

The authors declare that they have no conflict of interest.

\section{Ethics approval}

Approval was obtained from the Ethics Committee of Sun Yat-sen Memorial Hospital, Sun Yat-sen University (number, SYSEC-KY-KS-2021-225). Informed consent was exempt because of the retrospective nature of the study.

\section{Consent for publication}

Not applicable.

\section{Data and material}

Data are available from the corresponding author upon request.

\section{Acknowledgements}

Not applicable.

\section{Authors' contributions}

$J L$ designed the study, screened patients, collected and summarized the data and drafted the manuscript. YL reviewed and analyzed the radiological findings. XS and ZH reassessed the pathological examination. TY, LP and XJ screened patients, helped to collect and check data. LW designed the study and revised the manuscript. All authors read and approved the final manuscript.

\section{References}

1. Mahajan VS, Mattoo H, Deshpande V, Pillai SS, Stone JH. IgG4-related disease. Annu Rev Pathol. 2014;9:315-47.

2. Wallace ZS, Zhang Y, Perugino CA, Naden R, Choi HK, Stone JH; ACR/EULAR IgG4-RD Classification Criteria Clinical phenotypes of IgG4-related disease: an analysis of two international cross-sectional cohorts. Ann Rheum Dis. 2019;78(3):406-12.

3. Duvic C, Desrame J, Lévêque C, Nedelec G. Retroperitoneal fibrosis, sclerosing pancreatitis and bronchiolitis obliterans with organizing pneumonia. Nephrol Dial Transplant. 2004;19(9):2397-9.

4. Deshpande V, Zen Y, Chan JK, Yi EE, Sato Y, Yoshino T, et al. Consensus statement on the pathology of IgG4-related Mod Pathol. 2012;25(9):1181-92.

5. Inoue D, Zen Y, Abo H, Gabata T, Demachi H, Kobayashi T, et al. Immunoglobulin G4-related lung disease: CT findings with pathologic correlations. Radiology. 2009;251(1):260-70.

6. Kobayashi H, Shimokawaji T, Kanoh S, Motoyoshi K, Aida S. IgG4-positive pulmonary disease. J Thorac Imaging. 2007;22(4):360-2.

7. Sun X, Liu H, Feng R, Peng M, Hou X, Wang P, et al. Biopsy-proven IgG4-related lung disease. BMC Pulm Med. 2016; 25;16:20.

8. Kang J, Park S, Chae EJ, Song JS, Hwang HS, Kim SJ, et al. Long-term clinical course and outcomes of immunoglobulin G4-related lung disease. Respir Res. 2020;21(1):273.

9. Xie Y, Xiong A, Marion T, Liu Y. Lung nodules and IgG4 related disease: a single-center based experience. BMC Pulm 2020;20(1):218.

10. Bertoglio P, Viti A, Paiano S, Assante LR, Bogina GS, Pomari C, et al. IgG4-related disease: a new challenging diagnosis mimicking lung cancer. Interact Cardiovasc Thorac Surg. 2019;28(3):410-2.

11. Matsui S, Hebisawa A, Sakai F, Yamamoto H, Terasaki Y, Kurihara Y, et al. Immunoglobulin G4-related lung disease: clinicoradiological and pathological features. Respirology. 2013;18(3):480-7.

12. Chu ZG, Sheng B, Liu MQ, Lv FJ, Li Q, Ouyang Y. Differential Diagnosis of Solitary Pulmonary Inflammatory Lesions and Peripheral Lung Cancers with Contrast-enhanced Computed Tomography. Clinics (Sao Paulo). 2016;71(10):555-61.

13. Winer-Muram HT. The solitary pulmonary nodule. Radiology. 2006;239(1):34-49.

14. Heitzman ER, Markarian B, Raasch BN, Carsky EW, Lane EJ, Berlow ME. Pathways of tumor spread through the lung: radiologic correlations with anatomy and pathology. Radiology. 1982;144(1):3-14.

15. Yang H, Li J, Wang Y, Ye S, Li J. Distribution characteristics of elevated serum immunoglobulin G4 (IgG4) and its relationship with IgG4-related disease. Scand J Rheumatol. 2019;48(6):497-504.

16. Wang A, Fan J, Chen X, Wang S. An initial exploration for comprehensive assessment of IgG4-related lung disease: analyses on the cases enrolled from a systematic review. J Thorac Dis. 2018;10(3):1825-41.

17. Wen Z, Huang Y, Ling Z, Chen J, Wei X, Su R, et al. Lack of Efficacy of Combined Carbohydrate Antigen Markers for Lung Cancer Diagnosis. Dis Markers. 2020;2020:4716793.

18. Wang L, Wang D, Zheng G, Yang Y, Du L, Dong Z, e al. Clinical evaluation and therapeutic monitoring value of serum tumor markers in lung cancer. Int J Biol Markers. 2016;31(1):e80-7.

19. Okamura K, Takayama K, Izumi M, Harada T, Furuyama K, Nakanishi Y. Diagnostic value of CEA and CYFRA 21-1 tumor markers in primary lung cancer. Lung Cancer. 2013;80(1):45-9. 
20. Liu L, Teng J, Zhang L, Cong P, Yao Y, Sun G, et al. The Combination of the Tumor Markers Suggests the Histological Diagnosis of Lung Cancer. Biomed Res Int. 2017;2017:2013989.

21. Lanzillotta M, Mancuso G, Della-Torre E. Advances in the diagnosis and management of IgG4 related disease. BMJ. 2020;369:m1067.

\section{Figures}

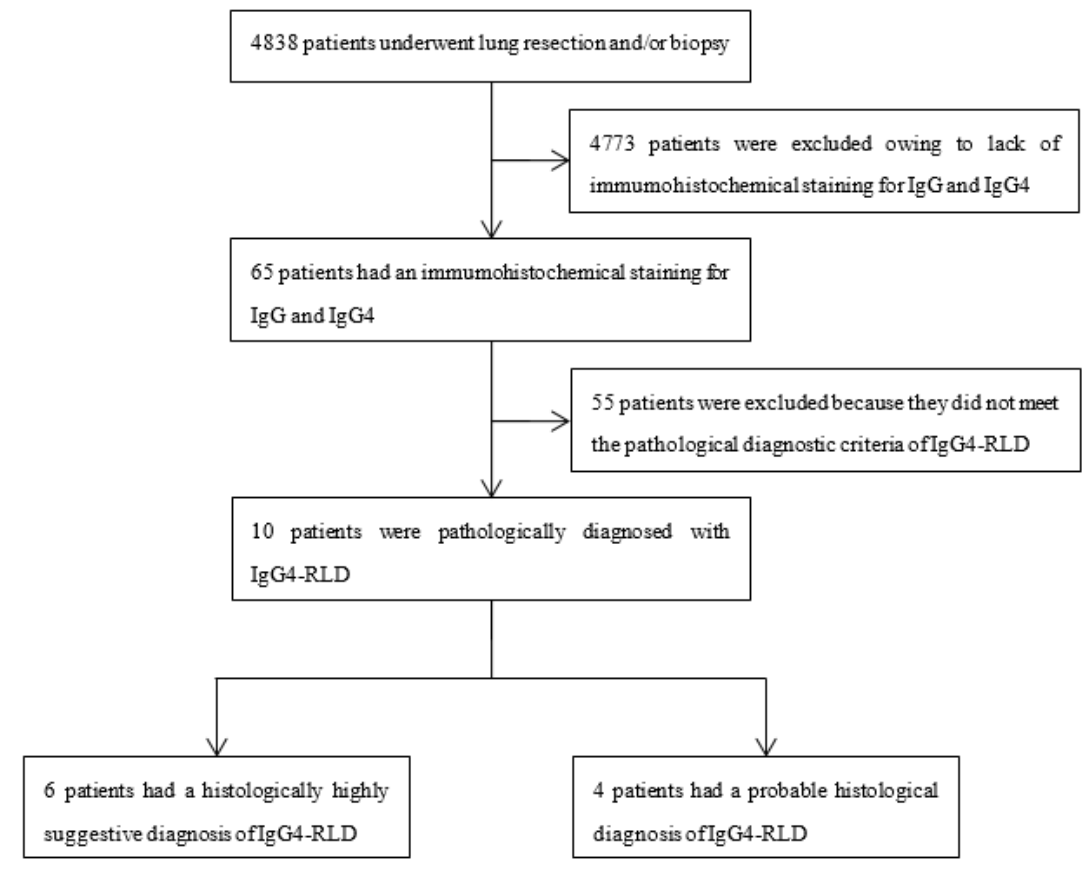

Figure 1

Flow diagram of the screening and diagnostic process for lgG4-related lung disease
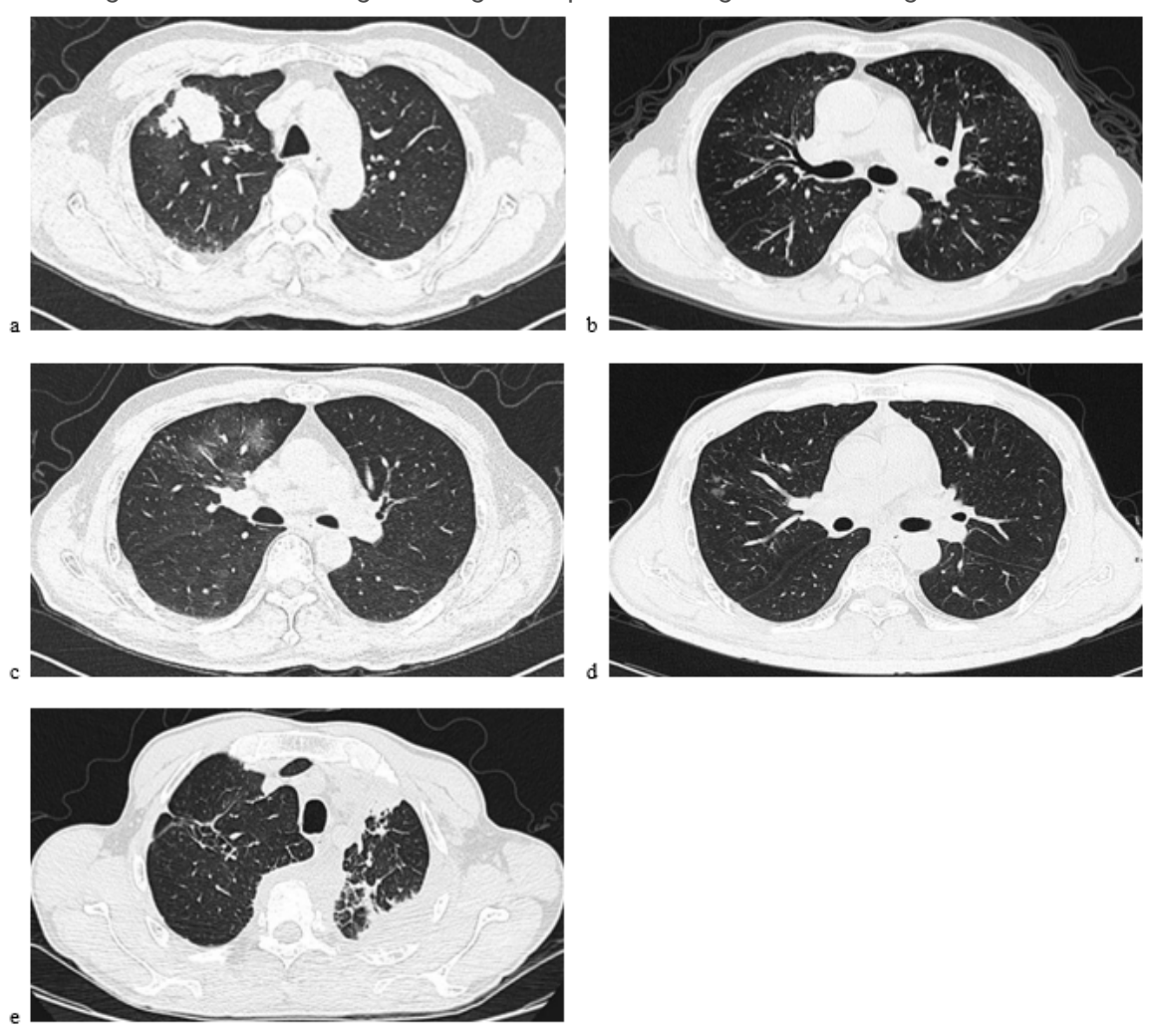

Figure 2 
Radiological manifestations of IgG4-related lung disease on chest computed tomography. a Mass with spiculated margin and pleural indentation lung is observed in the right. b Thickening of bronchovascular bundle and bronchiectasia are noted. $\mathrm{c}$ Diffuse ground-glass opacities are noted in the right lung. $d$ Round-shaped ground-glass opacities are observed in the right lung. e Alveolar consolidation and thickening of interlobular septa are shown in the left lung. 屏

\section{Figure 3}

Histopathological findings of IgG4-related lung disease. a Dense lymphoplasmocytic infiltration and storiform fibrosis are observed at low magnification (H\&E; $\times 40)$. b Abundant lymphoplasmic cell and fibrosis are noted at high magnification (H\&E; $\times 200)$. c Numerous IgG4+ plasma cells are demonstrated (IgG4 immunostaining; $\times 200)$. d Numerous IgG+ plasma cells are shown (IgG immunostaining; $\times 200)$. 